\title{
Selenium supplementation lowers insulin resistance and markers of cardio-metabolic risk in patients with congestive heart failure: a randomised, double-blind, placebo-controlled trial
}

\author{
Fariba Raygan $^{1}$, Milad Behnejad ${ }^{1}$, Vahidreza Ostadmohammadi ${ }^{2}$, Fereshteh Bahmani ${ }^{2}$, \\ Mohammad A. Mansournia ${ }^{3}$, Fatemeh Karamali ${ }^{2}$ and Zatollah Asemi $^{2 *}$ \\ ${ }^{1}$ Department of Cardiology, School of Medicine, Kashan University of Medical Sciences, Kashan, PO Box 8715988141, Iran \\ ${ }^{2}$ Research Center for Biochemistry and Nutrition in Metabolic Diseases, Kashan University of Medical Sciences, Kashan, PO \\ Box 8715988141, Iran \\ ${ }^{3}$ Department of Epidemiology and Biostatistics, School of Public Health, Tehran University of Medical Sciences, Tehran, PO \\ Box 141556446, Iran
}

(Submitted 24 October 2017 - Final revision received 25 December 2017 - Accepted 22 January 2018)

\section{Abstract}

This study was carried out to evaluate the effects of Se supplementation on metabolic profiles in patients with congestive heart failure (CHF). This randomised double-blind, placebo-controlled trial was performed among fifty-three subjects with CHF, aged $45-85$ years old. Subjects were randomly allocated into two groups to take either $200 \mu \mathrm{g} / \mathrm{d}$ of Se as Se yeast ( $n$ 26) or placebo $(n 27)$ for 12 weeks. Metabolic profiles were assessed at baseline and at the end of trial. Compared with the placebo, Se supplementation led to significant reductions in serum insulin ( -18.41 (sD 27.53$) v .+13.73$ (sD 23.63) pmol/1, $P<0 \cdot 001$ ), homoeostatic model of assessment for insulin resistance $(-1.01$ (sD 1.61$) v .+0 \cdot 55$ (sD 1.20$), P<0.001)$ and a significant increase in quantitative insulin sensitivity check index (QUICKI) (+0.007 (sD 0.03) $v .-0.01$ (sD 0.01), $P=0.007$ ). In addition, Se supplementation significantly decreased LDL-cholesterol $(-0.23$ (SD 0.29$) v .-0.04$ (sD 0.28$) \mathrm{mmol} / 1, P=0.03)$ and total-:HDL-cholesterol ratio ( -0.47 (sD 0.31$) v .-0.06$ (sD 0.42), $P<0.001$ ), and significantly increased HDL-cholesterol levels $(+0.18$ (sD 0.19$) v .+0.02$ (sD 0.13 ) mmol/1, $P=0.001)$ compared with the placebo. In addition, taking Se supplements was associated with a significant reduction in high-sensitivity C-reactive protein (hs-CRP) $(-1880 \cdot 8$ $(\mathrm{sD} 3437.5) v .+415.3(\mathrm{sD} 2116.5) \mathrm{ng} / \mathrm{ml}, P=0.01$ ), and a significant elevation in plasma total antioxidant capacity (TAC) (+30.9 (sD 118.0) $v .-187.9$ (sD 412.7$) \mathrm{mmol} / \mathrm{l}, P=0.004$ ) and total glutathione levels $(+33.7$ (sD 130.4) $v \cdot-39 \cdot 2$ (sD 132.8) $\mu \mathrm{mol} / 1, P=0 \cdot 003$ ) compared with the placebo. When we applied Bonferroni correction for multiple outcome testing, QUICKI $(P=0 \cdot 11)$, LDL-cholesterol $(P=0 \cdot 51)$, hs-CRP $(P=0 \cdot 17)$, TAC $(P=0 \cdot 06)$ and GSH $(P=0.05)$ became non-significant, and other metabolic profiles did not alter. Overall, our study supported that Se supplementation for 12 weeks to patients with CHF had beneficial effects on insulin metabolism and few markers of cardio-metabolic risk.

Key words: Selenium: Supplementation: Metabolic status: Congestive heart failure

Congestive heart failure $(\mathrm{CHF})$ is a clinical syndrome that is characterised by dyspnoea, orthopnoea, elevated jugular venous pressure and pulmonary congestion ${ }^{(1)}$. It is caused by a structural and/or functional cardiac abnormality resulting in decreased cardiac output and/or increased intracardiac pressures $^{(2)}$. The prevalence of $\mathrm{CHF}$ increases with age and is approximately ten per 1000 people for those older than 65 years $^{(3)}$. Patients with $\mathrm{CHF}$ are more prone to develop type 2 diabetes mellitus (T2DM), and thus approximately 20 to $35 \%$ of enrolled subjects have a clinical history of diabetes mellitus ${ }^{(4)}$. Several clinical studies have shown that high levels of oxidative stress markers and inflammatory cytokines may reflect the severity of $\mathrm{CHF}^{(5-7)}$.
A number of studies have documented a relationship between less severe Se deficiency or suboptimal Se levels and heart failure ${ }^{(8-9)}$. However, in another study, Se levels in CHF patients were similar to those of controls, and Se levels did not correlate with the degree of left ventricular dysfunction ${ }^{(10)}$. Prior studies have reported that Se deficiency is an accepted cause of reversible $\mathrm{CHF}^{(8,11)}$. However, in a Cochrane review ${ }^{(12)}$, Se supplementation was associated with a small non-significant increase in diabetes risk. In addition, in the above-mentioned study, there were no statistically significant effects of Se supplementation on all-cause mortality, CVD mortality, non-fatal CVD events or all CVD events ${ }^{(12)}$. On the other hand, the results of the selenium and vitamin E cancer prevention trial (SELECT)

Abbreviations: CHF, congestive heart failure; FPG, fasting plasma glucose; HOMA-IR, homoeostasis model of assessment-insulin resistance; hs-CRP, highsensitivity C-reactive protein; MDA, malondialdehyde; NO, nitric oxide; QUICKI, quantitative insulin sensitivity check index; T2DM, type 2 diabetes mellitus; TAC, total antioxidant capacity.

* Corresponding author: Z. Asemi, fax +98 31 55463377, email asemi_r@yahoo.com 
clearly do not support Se or vitamin E supplementation in adult life for primary prevention of cancer ${ }^{(13)}$. It has been suggested that Se may be involved in the deconditioning of skeletal and cardiac muscles and in CHF symptoms including fatigue and low exercise tolerance, rather than in ventricular dysfunction ${ }^{(14,15)}$. Some studies have reported the beneficial effects of Se supplementation on glycaemic control and biomarkers of inflammation and oxidative stress in patients with T2DM and $\mathrm{CHD}^{(16-18)}$.

This evidence may suggest the importance of Se in patients with CHF. However, whether Se has direct benefits on metabolic status in patients with CHF has not yet been assessed. In addition, data on the effects of Se on metabolic profiles in patients without CHF are conflicting. Therefore, the aim of this study was to examine the effects of Se supplementation on metabolic status among subjects with CHF.

\section{Methods \\ Participants}

This study is a randomised, double-blind, placebo-controlled trial, registered in the Iranian registry of clinical trials (http://www.irct.ir: IRCT2017053033941N2), conducted at a cardiology clinic affiliated to Kashan University of Medical Sciences (KaUMS), Kashan, Iran, between June 2017 and September 2017. The subjects were recruited between 1 June 2017 and 15 June 2017 from our Referral centre for CHF in Kashan, Iran. Then, the present trial was conducted among fifty-three participants with CHF from 16 June 2017 to 10 September 2017. Owing to the long duration of the administrative process, the registration number of our study seems retrospective; however, we received the formal ethics approval before beginning our study. Diagnosis of CHF was conducted based on the echocardiography method ${ }^{(19)}$. Those consuming Se supplements within the past 3 months, having an acute myocardial infarction within the past 3 months, having cardiac surgery within the past 3 months or significant renal or hepatic failure were not included in this study. This investigation was performed according to the principles of the Declaration of Helsinki, and the study protocol was approved by the ethics committee of KaUMS. All patients were informed about the aims and protocol of the study. Written informed consent was obtained from all subjects before the intervention.

\section{Study design}

At the onset of the study, to decrease potential confounding effects, all participants were subjected to stratified randomisation ${ }^{(20)}$ according to age, BMI, sex and the dosage and kind of medications. Thereafter, the participants in each block were randomly allocated into two treatment groups to take either $200 \mu \mathrm{g}$ of Se supplements as Se yeast ( $n$ 26) or placebo ( $n$ 27) per day for 12 weeks. Participants were asked to refrain from all other Se-containing supplements during the trial. Se and its placebos were purchased from Webber Naturals Pharmaceutical Company (lot no. LOT778342) and Barij Essence Pharmaceutical Company, respectively. Both Se supplements and placebo capsules had similar packaging, and patients and researchers were unaware of the content of the package until the end of study. Randomisation assignment was performed using computer-generated random numbers. Randomisation and allocation were concealed from the investigators and participants until the final analyses were completed. The randomised allocation sequence, enrolment of participants and allocation to interventions were conducted by a trained staff member at the cardiology clinic. Compliance with the intake of supplements and placebos was determined by examining the tablet containers. In addition, participants received a daily reminder message on their cell phones to take their supplements regularly. All participants completed 3-d dietary records (2 weekdays and 1 weekend day) at weeks 1, 5, 9 and 12 of the trial. To obtain nutrient intakes of participants according to 3-d food records, we applied the Nutritionist IV software (First Databank) adapted for the Iranian food pattern ${ }^{(21)}$.

\section{Assessment of anthropometric measures}

Weight (Seca) was assessed at baseline and after the 12-week intervention in cardiology clinic by a trained staff member. Height (Seca) was determined by a non-stretched tape measure to the nearest $0 \cdot 1 \mathrm{~cm}$. BMI was determined as weight in $\mathrm{kg}$ divided by height squared in $\mathrm{m}$.

\section{Outcomes}

Insulin levels and the homoeostasis model of assessment-insulin resistance (HOMA-IR) were considered as the primary outcome, and lipid profiles, biomarkers of inflammation and oxidative stress, and blood pressures were defined as the secondary outcomes. A volume of $10 \mathrm{ml}$ of fasting blood samples was drawn from the antecubital vein at the beginning and after the 12-week intervention at Kashan reference laboratory, Kashan, Iran. Blood was collected in two separate tubes: (1) one without EDTA to separate the serum, in order to quantify serum insulin, lipid profiles and high-sensitivity C-reactive protein (hs-CRP) concentrations, and (2) another one containing EDTA to examine plasma nitric oxide (NO) and biomarkers of oxidative stress. Fasting plasma glucose (FPG) levels were measured on the day of blood collection. Blood samples were immediately centrifuged (D-78532; Hettich) at $3500 \mathrm{rpm}$ for $10 \mathrm{~min}$ to separate the serum. The samples were then stored at $-80^{\circ} \mathrm{C}$ until analysis at the KaUMS reference laboratory. Serum insulin levels were assessed by the use of the ELISA kit (DiaMetra) with interand intra-assay CV of $3 \cdot 2-4 \cdot 5 \%$, respectively. HOMA-IR and the quantitative insulin sensitivity check index (QUICKI) were determined according to the standard formula ${ }^{(22)}$. Enzymatic kits (Pars Azmun) were applied to evaluate FPG, serum TAG, VLDL-, total-, LDL- and HDL-cholesterol levels. Serum hs-CRP levels were determined using a commercial ELISA kit (LDN) with interand intra-assay $\mathrm{CV}$ of $4 \cdot 5-6 \cdot 5 \%$, respectively. The plasma NO levels were determined using the Griess method ${ }^{(23)}$. Plasma total antioxidant capacity (TAC) levels by the method of ferricreducing antioxidant power developed by Benzie \& Strain ${ }^{(24)}$, total GSH using the method of Beutler \& Gelbart ${ }^{(25)}$ and malondialdehyde (MDA) concentrations by the thiobarbituric acid reactive substances spectrophotometric test ${ }^{(26)}$ were determined. CV for plasma TAC, GSH and MDA were lower than 5\%, respectively. All inter- and intra-assay CV for FPG and lipid values were $<5 \%$. Systolic (SBP) and diastolic blood pressure (DBP) was determined by means of a sphygmomanometer 
(ALPK2; Ningbo TianHou Import and Export Co., Ltd). Blood pressure values were reported in $\mathrm{mmHg}$.

\section{Statistical methods}

In the present study, we used the formula suggested for randomised clinical trials' sample size calculation. Type one $(\alpha)$ and type two errors $(\beta)$ were defined as 0.05 and 0.20 (power $=80 \%$ ), respectively. According to the previous trial ${ }^{(27)}$, we used 1.4 as the SD and 1.12 as the change in mean $(d)$ of HOMA-IR as a primary outcome in this formula. On the basis of the formula, we needed twenty-five subjects in each group; after allowing for five dropouts in each group, the final sample size was thirty persons in each group.

Multiple linear regression model was used to assess the intention-to-treat effect of treatment on study outcomes after adjusting for random confounding by the baseline values of outcome, age and BMI. Adjustment for age and BMI is necessary for two reasons: (i) to account for residual random confounding by age and BMI as stratified randomisation was only based on broad categories of these variables, and (ii) to obtain correct standard error for treatment effect ${ }^{(20,28-30)}$. Normality of residuals was assessed using normal probability plot and KolmogorovSmirnov test. Outcome log-transformation was used if model residual has non-normal distribution (QUICKI, NO, TAC, MDA, SBP and DBP). Bootstrapping was also used as a sensitivity analysis. Bonferroni correction (i.e. multiplying $P$ values by the number of tests) was used to account for multiple outcome testing. However, we note that our outcomes and thus statistical tests will tend to be positively correlated, and thus the Bonferroni procedure, which is based on independence of tests, is very conservative. The paired-samples $t$ test was used to detect within-group differences. $P$ values $<0.05$ were considered significant. All statistical analyses were performed by the Statistical Package for Social Science version 18 (SPSS Inc.).

\section{Results}

Among participants, four participants in the Se group (withdrawn owing to personal reasons $(n 4)$ ) and three participants in the placebo group (withdrawn owing to personal reasons ( $n$ 3)) did not complete the trial (Fig. 1). Finally, fifty-three participants (Se ( $n$ 26) and placebo ( $n$ 27)) completed the trial. The rate of compliance in our study ranged between 90 and $100 \%$ in both groups. No side effects were reported after Se supplementation in people with CHF throughout the study.

Mean age, height and weight and BMI at baseline and end of trial of study participants were not statistically different between Se and placebo groups (Table 1).

On the basis of the 3-d dietary records obtained throughout the intervention, no significant difference was observed between the two groups in terms of micronutrients and macronutrients (Table 2).

After the 12-week intervention, compared with the placebo, Se supplementation led to significant reductions in serum insulin levels (-18.41 (sD 27.53) v. +13.73 (sD 23.63) pmol/1, $P<0.001)$, HOMA-IR (-1.01 (SD 1.61) v. +0.55 (sD 1.20), $P<0.001)$ and a significant increase in QUICKI ( +0.007 (SD 0.03) $v$. $-0 \cdot 01$ (sD 0.01), $P=0 \cdot 007$ ) (Table 3). In addition, Se supplementation significantly decreased serum LDL-cholesterol levels $(-0.23$ (sD 0.29) $v$. -0.04 (sD 0.28) $\mathrm{mmol} / \mathrm{l}, P=0.03)$ and total-:HDLcholesterol ratio $(-0.47$ (SD 0.31) v. -0.06 (SD 0.42), $P<0.001$ ), and significantly increased HDL-cholesterol levels (+0.18 (SD 0.19) $v$. $+0.02(\mathrm{sD} 0 \cdot 13) \mathrm{mmol} / \mathrm{l}, P=0 \cdot 001)$ compared with the placebo.

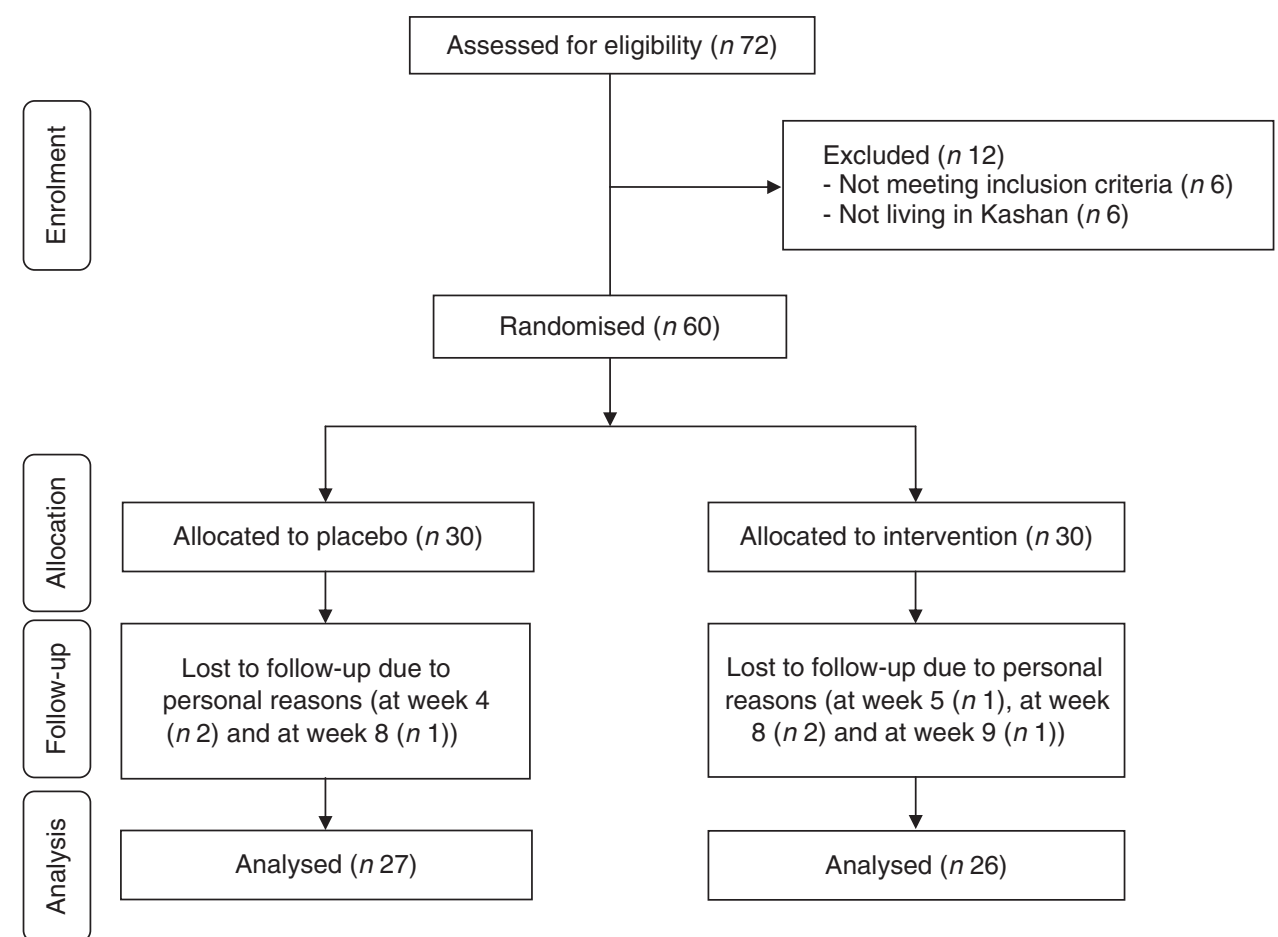

Fig. 1. Summary of patient flow diagram. 
Table 1. General characteristics of study participants (Mean values and standard deviations; numbers and percentages)

\begin{tabular}{|c|c|c|c|c|c|}
\hline & \multicolumn{2}{|c|}{$\begin{array}{l}\text { Placebo group } \\
\quad(n 27)\end{array}$} & \multicolumn{2}{|c|}{$\begin{array}{l}\text { Se group } \\
(n 26)\end{array}$} & \multirow[b]{2}{*}{$P^{\star}$} \\
\hline & Mean & SD & Mean & SD & \\
\hline Age (years) & 68.5 & $7 \cdot 7$ & 70.7 & $10 \cdot 3$ & 0.37 \\
\hline Height (cm) & $162 \cdot 1$ & 11.0 & $160 \cdot 5$ & $10 \cdot 7$ & 0.59 \\
\hline Weight at study baseline $(\mathrm{kg})$ & 67.9 & $7 \cdot 1$ & $65 \cdot 8$ & 9.4 & 0.36 \\
\hline Weight at the end of trial $(\mathrm{kg})$ & $67 \cdot 9$ & 7.9 & $66 \cdot 2$ & 9.5 & 0.46 \\
\hline Weight change (kg) & 0.005 & 1.4 & 0.3 & $1 \cdot 3$ & 0.35 \\
\hline BMI at study baseline $\left(\mathrm{kg} / \mathrm{m}^{2}\right)$ & $26 \cdot 2$ & 4.3 & $25 \cdot 7$ & 4.1 & 0.69 \\
\hline BMl at the end of trial $\left(\mathrm{kg} / \mathrm{m}^{2}\right)$ & $26 \cdot 2$ & 4.6 & $25 \cdot 84$ & $4 \cdot 0$ & 0.77 \\
\hline BMI change $\left(\mathrm{kg} / \mathrm{m}^{2}\right)$ & 0.01 & 0.5 & 0.1 & 0.5 & 0.46 \\
\hline \multicolumn{6}{|l|}{ Sex } \\
\hline Female & \multirow{2}{*}{\multicolumn{2}{|c|}{19}} & & & $0.92 \dagger$ \\
\hline$n$ & & & \multicolumn{2}{|c|}{18} & \\
\hline$\%$ & \multicolumn{2}{|c|}{$70 \cdot 7$} & \multicolumn{2}{|c|}{$69 \cdot 2$} & \\
\hline \multicolumn{6}{|l|}{ Male } \\
\hline$n$ & \multirow{2}{*}{\multicolumn{2}{|c|}{$\begin{array}{c}8 \\
29.6\end{array}$}} & \multirow{2}{*}{\multicolumn{2}{|c|}{8}} & \\
\hline$\%$ & & & & & \\
\hline
\end{tabular}

* Obtained from independent $t$ test.

t Obtained from Pearson's $x^{2}$ test.

Table 2. Dietary intakes of study participants at weeks $1,5,9$ and 12 of the study

(Mean values and standard deviations)

\begin{tabular}{|c|c|c|c|c|c|}
\hline & \multicolumn{2}{|c|}{ Placebo group ( $n$ 27) } & \multicolumn{2}{|c|}{ Se group ( $n$ 26) } & \multirow[b]{2}{*}{$P^{\star}$} \\
\hline & Mean & SD & Mean & SD & \\
\hline Energy $(\mathrm{kJ} / \mathrm{d})$ & 9447 & 1092 & 9222 & 1393 & 0.51 \\
\hline Energy $(\mathrm{kcal} / \mathrm{d})$ & 2258 & 261 & 2204 & 333 & 0.51 \\
\hline Carbohydrates $(\mathrm{g} / \mathrm{d})$ & 309.1 & 53.1 & 301.4 & 67.6 & 0.64 \\
\hline Protein $(\mathrm{g} / \mathrm{d})$ & $81 \cdot 7$ & $14 \cdot 4$ & 83.6 & $17 \cdot 6$ & 0.66 \\
\hline Fat $(g / d)$ & $80 \cdot 9$ & 9.8 & $77 \cdot 3$ & 14.5 & 0.29 \\
\hline SFA $(g / d)$ & 24.9 & $4 \cdot 3$ & 24.6 & $5 \cdot 2$ & 0.80 \\
\hline MUFA (g/d) & $25 \cdot 0$ & 5.5 & $24 \cdot 4$ & $6 \cdot 0$ & 0.71 \\
\hline PUFA $(\mathrm{g} / \mathrm{d})$ & $22 \cdot 3$ & 4.4 & $22 \cdot 2$ & 4.5 & 0.97 \\
\hline Cholesterol $(\mathrm{mg} / \mathrm{d})$ & $242 \cdot 3$ & $132 \cdot 1$ & $262 \cdot 3$ & 87.2 & 0.51 \\
\hline $\operatorname{TDF}(\mathrm{g} / \mathrm{d})$ & $19 \cdot 3$ & 4.9 & 19.2 & 4.8 & 0.92 \\
\hline $\operatorname{Se}(\mu \mathrm{g} / \mathrm{d})$ & $56 \cdot 3$ & 7.6 & $55 \cdot 6$ & $10 \cdot 1$ & 0.78 \\
\hline$M g(m g / d)$ & $268 \cdot 1$ & $50 \cdot 3$ & 255.9 & $49 \cdot 4$ & 0.37 \\
\hline $\mathrm{Mn}(\mathrm{mg} / \mathrm{d})$ & $2 \cdot 1$ & 0.7 & 2.0 & 0.8 & 0.56 \\
\hline $\mathrm{Fe}(\mathrm{mg} / \mathrm{d})$ & $14 \cdot 3$ & 3.0 & $13 \cdot 2$ & 3.1 & 0.20 \\
\hline $\mathrm{Zn}(\mathrm{mg} / \mathrm{d})$ & $10 \cdot 2$ & $2 \cdot 3$ & $10 \cdot 4$ & 2.8 & 0.80 \\
\hline $\mathrm{Ca}(\mathrm{mg} / \mathrm{d})$ & 945.5 & $438 \cdot 8$ & 811.9 & 474.6 & 0.29 \\
\hline$P(\mathrm{mg} / \mathrm{d})$ & $953 \cdot 3$ & 431.6 & 838.3 & $487 \cdot 2$ & 0.36 \\
\hline $\mathrm{Cr}(\mu \mathrm{g} / \mathrm{d})$ & $32 \cdot 0$ & $10 \cdot 9$ & 27.5 & 10.4 & 0.36 \\
\hline Vitamin $E(\mathrm{mg} / \mathrm{d})$ & $12 \cdot 2$ & 1.3 & $12 \cdot 0$ & 1.2 & 0.65 \\
\hline Vitamin C $(\mathrm{mg} / \mathrm{d})$ & $75 \cdot 1$ & $10 \cdot 4$ & 73.1 & 9.7 & 0.56 \\
\hline
\end{tabular}

TDF, total dietary fibre.

* Obtained from independent-samples $t$ test.

In addition, taking Se supplements was associated with a significant reduction in hs-CRP $(-1880.8$ (sD 3437.5) $v$. $+415.3(\mathrm{SD} 2116.5) \mathrm{ng} / \mathrm{ml}$, $P=0.01)$, and a significant elevation in plasma TAC (+30.9 (SD 118.0) v. $-187.9(\mathrm{SD} 412.7) \mathrm{mmol} / \mathrm{l}, P=0.004)$ and GSH levels $(+33.7$ (SD 130.4) $v .-39 \cdot 2$ (SD 132.8) $\mu \mathrm{mol} / 1, P=0.003$ ) compared with the placebo. When we applied Bonferroni correction, QUICKI $(P=0.11)$, LDL-cholesterol $(P=0.51)$, hs-CRP $(P=0 \cdot 17)$, TAC $(P=0.06)$ and GSH $(P=0.05)$ became non-significant, and other metabolic profiles did not alter. Se supplementation did not improve other metabolic profiles. Using bootstrapping analyses, our findings did not change. Within-group changes demonstrated a significant decrease of FPG $(P=0.04)$, serum insulin $(P=0.002)$, HOMA-IR $(P=0.004)$, LDL- $(P<0 \cdot 001)$, total-:HDL-cholesterol ratio $(P<0 \cdot 001)$, hs-CRP $(P=0.01)$ and SBP $(P=0.04)$, and a significant increase of serum HDL-cholesterol levels $(P<0.001)$ in the Se group. In addition, within-group changes revealed a significant increase of serum insulin $(P=0.006)$ and HOMA-IR $(P=0.02)$, and a significant decrease of QUICKI $(P=0.001)$, plasma NO $(P=0.02)$ and TAC levels $(P=0.02)$ in the placebo group.

\section{Discussion}

In the present study, which to our knowledge is the first report of Se supplementation in patients with CHF, we evaluated the effects of Se supplementation on markers of insulin metabolism, lipid profiles, biomarkers of inflammation and oxidative stress. The major finding was that Se supplementation improved insulin metabolism, decreased serum LDL-cholesterol, total-/ HDL-cholesterol, hs-CRP and increased serum HDL-cholesterol, plasma TAC and GSH concentrations in patients with CHF, but did not improve other metabolic profiles. When we applied Bonferroni correction for multiple outcome testing, QUICKI, LDL-cholesterol, hs-CRP, TAC and GSH became non-significant, and other metabolic profiles did not alter. However, some studies have reported no beneficial effects of Se supplementation on the incidence of diabetes and metabolic profiles in patients with metabolic diseases. Stranges et al. ${ }^{(31)}$ reported that Se supplementation increased T2DM incidence in a randomised controlled trial. In the above-mentioned study, an exposureresponse gradient was seen across tertiles of baseline Se levels, with a statistically significantly increased risk for T2DM in the highest tertile of baseline Se levels ${ }^{(31)}$. Such an association has also been documented in a number of observational studies, generally with cross-sectional and prospective design. In a study by Galan-Chilet et $a l{ }^{(32)}$, Se levels were positively associated with prevalent and incident diabetes. Moreover, in US adults, high Se levels were associated with higher prevalence of diabetes and higher FPG and glycosylated $\mathrm{Hb}$ levels ${ }^{(33)}$. The effect of Se supplementation at dosages of 100, 200 and $300 \mu \mathrm{g} /$ $\mathrm{d}$ for 6 months and 5 years to elderly population on plasma cholesterol concentrations or its sub-fractions did not differ significantly from the placebo ${ }^{(34)}$. Se supplementation at a dosage of $100 \mu \mathrm{g} / \mathrm{d}$ as Se yeast in pregnant women for the last 6 months of pregnancy was also associated with increased cordblood TAG levels, although total-, LDL- and HDL-cholesterol levels did not change significantly ${ }^{(35)}$. In addition, the levels found in the previous study were higher than those reported in non-pregnant women from other parts of $\operatorname{Iran}^{(36,37)}$. The differences in circulating Se levels are probably owing to the regional and geographic variability in the Se content of soil and plant foods ${ }^{(38)}$. Different study designs, different dosages of Se used, potential differences in Se status of the participants in different studies along with characteristics of study participants might provide some reasons for discrepant findings. In addition, we could not explore the possibility that the different results might relate to genetic differences. Alternatively, there may have been differences in the intake of dietary macronutrients and micronutrients between different populations that could have modified the effects of additional Se on metabolic profiles. 
Table 3. Metabolic profiles, biomarkers of inflammation and oxidative stress at baseline and 12 weeks after the intervention in patients with congestive heart failure (Mean values and standard deviations)

\begin{tabular}{|c|c|c|c|c|c|c|c|c|c|c|c|c|c|c|c|c|}
\hline & \multicolumn{7}{|c|}{ Placebo group ( $n 27)$} & \multicolumn{7}{|c|}{ Se group $(n 26)$} & \multirow[b]{3}{*}{$P \dagger$} & \multirow[b]{3}{*}{$P \neq$} \\
\hline & \multicolumn{2}{|c|}{ Week 0} & \multicolumn{2}{|c|}{ Week 12} & \multicolumn{2}{|c|}{ Change } & \multirow[b]{2}{*}{$P^{\star}$} & \multicolumn{2}{|c|}{ Week 0} & \multicolumn{2}{|c|}{ Week 12} & \multicolumn{2}{|c|}{ Change } & \multirow[b]{2}{*}{$P^{*}$} & & \\
\hline & Mean & SD & Mean & SD & Mean & SD & & Mean & SD & Mean & SD & Mean & SD & & & \\
\hline $\mathrm{FPG}(\mathrm{mmol} / \mathrm{l})$ & 6.85 & 1.92 & 6.92 & 2.33 & 0.07 & 1.07 & 0.76 & $6 \cdot 18$ & 1.55 & 5.76 & 1.23 & -0.42 & 1.01 & 0.04 & 0.05 & 0.85 \\
\hline Insulin (pmol//) & 69.88 & 38.49 & 83.62 & 37.81 & 13.73 & 23.63 & 0.006 & 79.33 & 58.94 & 60.92 & 40.79 & -18.41 & 27.53 & 0.002 & $<0.001$ & $<0.001$ \\
\hline HOMA-IR & 3.66 & 2.67 & $4 \cdot 21$ & 2.46 & 0.55 & $1 \cdot 20$ & 0.02 & 4.05 & 3.92 & 3.04 & $2 \cdot 61$ & -1.01 & 1.61 & 0.004 & $<0.001$ & $<0.001$ \\
\hline QUICKI & 0.32 & 0.03 & 0.31 & 0.01 & -0.01 & 0.01 & 0.001 & 0.33 & 0.05 & 0.34 & 0.04 & 0.007 & 0.03 & 0.13 & 0.007 & 0.11 \\
\hline TAG $(\mathrm{mmol} / \mathrm{l})$ & 1.50 & 0.38 & 1.53 & 0.39 & 0.03 & 0.19 & 0.51 & 1.56 & 0.68 & 1.55 & 0.68 & -0.01 & 0.28 & 0.88 & 0.72 & $>0.99$ \\
\hline VLDL-cholesterol $(\mathrm{mmol} / \mathrm{l})$ & 0.69 & 0.17 & 0.70 & 0.18 & 0.01 & 0.08 & 0.51 & 0.71 & 0.31 & 0.71 & 0.31 & -0.002 & 0.12 & 0.88 & 0.72 & $>0.99$ \\
\hline Total-cholesterol $\left(\mathrm{mmol} / \mathrm{l}^{\prime}\right.$ & 4.01 & 0.91 & 4.01 & 0.99 & -0.0002 & 0.30 & 0.99 & 3.80 & 0.91 & 3.76 & 1.00 & -0.04 & 0.31 & 0.47 & 0.77 & $>0.99$ \\
\hline LDL-cholesterol (mmol/l) & $2 \cdot 16$ & 0.80 & $2 \cdot 12$ & 0.87 & -0.04 & 0.28 & 0.55 & 1.95 & 0.71 & 1.72 & 0.75 & -0.23 & 0.29 & $<0.001$ & 0.03 & 0.51 \\
\hline HDL-cholesterol (mmol/l) & 1.16 & 0.22 & $1 \cdot 18$ & 0.24 & 0.02 & 0.13 & 0.42 & $1 \cdot 13$ & 0.24 & 1.32 & 0.38 & 0.18 & 0.19 & $<0.001$ & 0.001 & 0.01 \\
\hline Total-:HDL-cholesterol ratio & 3.53 & 0.96 & 3.47 & 0.96 & -0.06 & 0.42 & 0.41 & 3.41 & 0.75 & 2.94 & 0.78 & -0.47 & 0.31 & $<0.001$ & $<0.001$ & $<0.001$ \\
\hline hs-CRP (ng/ml) & $3092 \cdot 3$ & $3148 \cdot 3$ & 3507.5 & 3483.8 & $415 \cdot 3$ & 2116.5 & 0.31 & 4138.5 & $3226 \cdot 3$ & 2257.7 & 1947.5 & $-1880 \cdot 8$ & 3437.5 & 0.01 & 0.01 & 0.17 \\
\hline $\mathrm{NO}(\mu \mathrm{mol} / \mathrm{l})$ & 35.6 & 7.8 & 32.8 & 8.5 & $-2 \cdot 8$ & $6 \cdot 1$ & 0.02 & $34 \cdot 2$ & 1.6 & 33.6 & 1.1 & -0.6 & 1.8 & 0.07 & 0.18 & $>0.99$ \\
\hline TAC $(\mathrm{mmol} / \mathrm{l})$ & 1183.5 & 344.5 & 995.6 & 356.9 & -187.9 & $412 \cdot 7$ & 0.02 & $1096 \cdot 2$ & 58.6 & $1127 \cdot 1$ & 134.2 & 30.9 & $118 \cdot 0$ & 0.19 & 0.004 & 0.06 \\
\hline $\mathrm{GSH}(\mu \mathrm{mol} / \mathrm{l})$ & 519.6 & $125 \cdot 6$ & 480.4 & 160.5 & -39.2 & 132.8 & 0.13 & 597.4 & 77.0 & 631.2 & $107 \cdot 1$ & 33.7 & $130 \cdot 4$ & 0.19 & 0.003 & 0.05 \\
\hline MDA $(\mu \mathrm{mol} / \mathrm{l})$ & $2 \cdot 9$ & $1 \cdot 1$ & 3.0 & $1 \cdot 1$ & 0.1 & 0.6 & 0.68 & $2 \cdot 5$ & 0.2 & $2 \cdot 4$ & 0.2 & -0.1 & 0.2 & 0.15 & 0.34 & $>0.99$ \\
\hline $\mathrm{SBP}(\mathrm{mmHg})$ & 126.6 & 14.1 & $122 \cdot 0$ & 14.4 & $-4 \cdot 6$ & 14.5 & 0.11 & 131.9 & $14 \cdot 1$ & $127 \cdot 7$ & $12 \cdot 5$ & $-4 \cdot 2$ & $10 \cdot 3$ & 0.04 & 0.33 & $>0.99$ \\
\hline DBP $(\mathrm{mmHg})$ & $79 \cdot 2$ & 9.7 & $77 \cdot 3$ & 9.6 & -1.9 & 9.9 & 0.32 & 88.1 & $9 \cdot 8$ & $86 \cdot 1$ & $9 \cdot 0$ & -1.9 & 8.5 & 0.25 & 0.09 & $>0.99$ \\
\hline
\end{tabular}

FPG, fasting plasma glucose; HOMA-IR, homoeostasis model of assessment-estimated insulin resistance; QUICKI, quantitative insulin sensitivity check index; hs-CRP, high-sensitivity C-reactive protein; NO, nitric oxide; TAC, total antioxidant capacity; MDA, malondialdehyde; SBP, systolic blood pressure; DBP, diastolic blood pressure.

Obtained from paired-samples $t$ test.

† Obtained from multiple regression model (adjusted for baseline values of each biochemical variables, age and baseline BMI).

$\ddagger$ Obtained from multiple regression model and corrected using Bonferroni correction $(P$ value $\times 17)$. 
It must be kept in mind that there is a small possibility of selection bias in our study. To decrease potential confounding effects, all participants were categorised according to age, BMI, sex and the dosage and kind of medications at the onset of the study. Then, participants in each category were randomly allocated into two treatment groups to take either Se supplements or placebo. We believe that further studies are needed to confirm our findings.

The present study evidenced that Se supplementation for 12 weeks to patients with CHF resulted in a significant decrease in serum insulin concentrations and HOMA-IR, LDL-cholesterol, total-:HDL-cholesterol and a significant elevation in serum HDLcholesterol and QUICKI compared with the placebo, but had no significant effect on FPG and other lipid concentrations. When we applied Bonferroni correction for multiple outcome testing, QUICKI and LDL-cholesterol became non-significant, and other metabolic profiles did not alter. Earlier, few studies have reported that $\mathrm{CHF}$ was correlated with lower Se and Zn concentrations $^{(9,14)}$. It has been suggested that Se element may be involved in the cardiac muscles and in CHF symptoms including fatigue and low exercise tolerance, rather than in ventricular dysfunction $^{(14,15)}$. Some observational studies, as well as Se supplementation trials, in which the association between Se levels/Se intake and the risk of diabetes or glycaemic control has been evaluated, generated inconsistent results ${ }^{(39,40)}$. On the other hand, animal studies demonstrated that Se supplementation may lead to hyperinsulinaemia and glucose intolerance ${ }^{(41)}$. Few studies have assessed the effects of Se supplementation on glycaemic control and lipid profiles in patients with T2DM, CHD and metabolic diseases. In a meta-analysis study, we have previously demonstrated that Se administration to patients with metabolic diseases improved insulin levels and QUICKI, but did not influence HOMA-IR and lipid profiles ${ }^{(18)}$. Taking Se supplements at a dose of $200 \mu \mathrm{g} / \mathrm{d}$ for 6 weeks resulted in a significant reduction in serum insulin levels and HOMA-IR in subjects with central obesity ${ }^{(42)}$. Se supplementation for 4 months to Sprague-Dawley rats lowered TAG levels, whereas other lipid profiles remained unchanged ${ }^{(43)}$. Insulin resistance and hyperinsulinaemia were correlated with inflammation, oxidative stress, cardiac remodelling and endothelial dysfunction that lead to decreasing endothelial NO synthase expression, which in turn result in an increase in vascular tone ${ }^{(44)}$. Furthermore, lipid accumulation in the heart, by the production of toxic intermediate products and derangement of insulin and oxidative pathways, determines conditions known as lipotoxicity and lipoapoptosis that impair cardiac function and promote $\mathrm{CHF}^{(45)}$. Se administration may improve insulin metabolism by inhibiting the expression of cyclo-oxygenase-2 and P-selectin ${ }^{(46)}$ and suppressing the production of inflammatory markers including TNF- $\alpha$ and $\mathrm{IL}^{(47)}$.

We found that Se supplementation in patients with $\mathrm{CHF}$ resulted in a significant decrease in serum hs-CRP, and a significant increase in plasma TAC and GSH levels, but did not change plasma NO and MDA levels compared with the placebo. When we applied Bonferroni correction for multiple outcome testing, hs-CRP, TAC and GSH became non-significant, and other metabolic profiles did not alter. Supplementation with combined Se and coenzyme Q10 for 48 months to elderly individuals decreased CRP and sP-selectin levels ${ }^{(48)}$. Previous studies in comparable groups have demonstrated that Se administration decreased $\mathrm{NF}-\kappa \mathrm{B}$ activation, and down-regulated gene expression related to inflammatory cytokines in macrophages ${ }^{(49,50)}$. High-dose Se supplementation for $14 \mathrm{~d}$ to sepsis patients did not decrease CRP levels, but Se levels correlated with glutathione peroxidase and prealbumin concentrations ${ }^{(51)}$. In another study, Se intake significantly increased MDA and hydroxyl radical levels in the lens of naphthalene-treated rats ${ }^{(52)}$. Increased systemic inflammation is a critical element underlying the pathophysiology of $\mathrm{CHF}$, contributing to myocardial remodelling and peripheral vascular damage ${ }^{(53)}$. Moreover, increased oxidative stress markers correlate positively with clinical parameters of $\mathrm{CHF}$ and their high concentrations are a poor prognostic factor in patients with $\mathrm{CHF}^{(54)}$. Therefore, decreased biomarkers of inflammation and oxidative stress by Se may decrease complications related to CHF.

The present study had a few limitations. For shortage of funding, we did not verify plasma or urine Se levels in the supplement and placebo groups. Whether Se supplementation is beneficial or detrimental depends on Se status before supplementation of this element; Se status was not measured in this study. Few studies have reported that high Se levels were associated with low overall mortality ${ }^{(55,56)}$. Increasing serum Se levels up to about $130 \mu \mathrm{g} / \mathrm{l}$ were associated with reduced mortality $^{(55)}$. In the 9-year longitudinal Epidemiology of Vascular Ageing (EVA) study, plasma se levels at baseline (mean $87 \mu \mathrm{g} / \mathrm{l}$ ) were associated with increased overall and cancer mortality ${ }^{(56)}$. In the Baltimore Women's Health and Aging Study, low Se level was a significant independent predictor of all-cause 5-year mortality in older women living in the community ${ }^{(57)}$. Therefore, this should be considered in our interpretation. In addition, we did not evaluate gene expression related to insulin, lipid and inflammation.

Overall, our study supported that Se supplementation for 12 weeks to patients with CHF had beneficial effects on insulin metabolism and few markers of cardio-metabolic risk.

\section{Acknowledgements}

The authors would like to thank the staff of Shahid Beheshti Clinic for their assistance in this project.

The present study was funded by a grant from the Vicechancellor for Research, KaUMS, Iran.

Z. A. contributed in conception, design, statistical analysis and drafting of the manuscript. F. R., M. B., V. O., F. B., M. A. M. and F. K. contributed in data collection and manuscript drafting. Z. A. supervised the study.

The authors declare that there are no conflicts of interest.

\section{References}

1. Kurmani S \& Squire I (2017) Acute heart failure: definition, classification and epidemiology. Curr Heart Fail Rep 14, 385-392.

2. Ponikowski P, Voors AA, Anker SD, et al. (2016) 2016 ESC Guidelines for the diagnosis and treatment of acute and chronic heart failure: The Task Force for the diagnosis and treatment of acute and chronic heart failure of the European 
Society of Cardiology (ESC). Developed with the special contribution of the Heart Failure Association (HFA) of the ESC. Eur J Heart Fail 18, 891-975.

3. Lloyd-Jones DM, Larson MG, Leip EP, et al. (2002) Lifetime risk for developing congestive heart failure: the Framingham Heart Study. Circulation 106, 3068-3072.

4. Horowitz JD \& Kennedy JA (2006) Time to address the cardiac metabolic 'triple whammy' ischemic heart failure in diabetic patients. I Am Coll Cardiol 48, 2232-2234.

5. Maack C \& Bohm M (2011) Targeting mitochondrial oxidative stress in heart failure throttling the afterburner. I Am Coll Cardiol 58, 83-86.

6. Diaz-Velez CR, Garcia-Castineiras S, Mendoza-Ramos E, et al. (1996) Increased malondialdehyde in peripheral blood of patients with congestive heart failure. Am Heart J 131, 146-152.

7. Aukrust P, Yndestad A, Damas JK, et al. (2004) Inflammation and chronic heart failure-potential therapeutic role of intravenous immunoglobulin. Autoimmun Rev 3, 221-227.

8. Cenac A, Simonoff M \& Djibo A (1996) Nutritional status and plasma trace elements in peripartum cardiomyopathy. A comparative study in Niger. J Cardiovasc Risk 3, 483-487.

9. Kosar F, Sahin I, Taskapan C, et al. (2006) Trace element status (Se, $\mathrm{Zn}, \mathrm{Cu}$ ) in heart failure. Anadolu Kardiyol Derg $\mathbf{6}$, 216-220.

10. Ghaemian A, Salehifar E, Shiraj H, et al. (2012) A comparison of selenium concentrations between congestive heart failure patients and healthy volunteers. J Tehran Heart Cent $\mathbf{7}$, 53-57.

11. Ge K \& Yang G (1993) The epidemiology of selenium deficiency in the etiological study of endemic diseases in China. Am J Clin Nutr 57, 259S-263S.

12. Rees K, Hartley L, Day C, et al. (2013) Selenium supplementation for the primary prevention of cardiovascular disease. Cochrane Database Syst Rev, issue 1, CD009671.

13. Lippman SM, Klein EA, Goodman PJ, et al. (2009) Effect of selenium and vitamin $\mathrm{E}$ on risk of prostate cancer and other cancers: the Selenium and Vitamin E Cancer Prevention Trial (SELECT). JAMA 301, 39-51.

14. de Lorgeril M, Salen P, Accominotti M, et al. (2001) Dietary and blood antioxidants in patients with chronic heart failure. Insights into the potential importance of selenium in heart failure. Eur J Heart Fail 3, 661-669.

15. de Lorgeril M \& Salen P (2006) Selenium and antioxidant defenses as major mediators in the development of chronic heart failure. Heart Fail Rev 11, 13-17.

16. Farrokhian A, Bahmani F, Taghizadeh M, et al. (2016) Selenium supplementation affects insulin resistance and serum hs-CRP in patients with type 2 diabetes and coronary heart disease. Horm Metab Res 48, 263-268.

17. Ju W, Li X, Li Z, et al. (2017) The effect of selenium supplementation on coronary heart disease: a systematic review and meta-analysis of randomized controlled trials. I Trace Elem Med Biol 44, 8-16.

18. Tabrizi R, Akbari M, Moosazadeh M, et al. (2017) The effects of selenium supplementation on glucose metabolism and lipid profiles among patients with metabolic diseases: a systematic review and meta-analysis of randomized controlled trials. Horm Metab Res 49, 826-830.

19. Dickstein K, Cohen-Solal A, Filippatos G, et al. (2008) ESC guidelines for the diagnosis and treatment of acute and chronic heart failure 2008: the Task Force for the diagnosis and treatment of acute and chronic heart failure 2008 of the European Society of Cardiology. Developed in collaboration with the Heart Failure Association of the ESC (HFA) and endorsed by the European Society of Intensive Care Medicine (ESICM). Eur J Heart Fail 10, 933-989.
20. Mansournia MA, Hernan MA \& Greenland S (2013) Matched designs and causal diagrams. Int J Epidemiol 42, 860-869.

21. Azar M \& Sarkisian E (1980) Food Composition Table of Iran. Tehran: National Nutrition and Food Research Institute, Shaheed Beheshti University.

22. Pisprasert V, Ingram KH, Lopez-Davila MF, et al. (2013) Limitations in the use of indices using glucose and insulin levels to predict insulin sensitivity: impact of race and gender and superiority of the indices derived from oral glucose tolerance test in African Americans. Diabetes Care 36, 845-853.

23. Tatsch E, Bochi GV, Pereira Rda S, et al. (2011) A simple and inexpensive automated technique for measurement of serum nitrite/nitrate. Clin Biochem 44, 348-350.

24. Benzie IF \& Strain JJ (1996) The ferric reducing ability of plasma (FRAP) as a measure of 'antioxidant power': the FRAP assay. Anal Biochem 239, 70-76.

25. Beutler E \& Gelbart T (1985) Plasma glutathione in health and in patients with malignant disease. J Lab Clin Med 105, 581-584.

26. Janero DR (1990) Malondialdehyde and thiobarbituric acidreactivity as diagnostic indices of lipid peroxidation and peroxidative tissue injury. Free Radic Biol Med 9, 515-540.

27. Bahmani F, Kia M, Soleimani A, et al. (2016) Effect of selenium supplementation on glycemic control and lipid profiles in patients with diabetic nephropathy. Biol Trace Elem Res 172, 282-289.

28. Mansournia MA \& Altman DG (2018) Invited commentary: methodological issues in the design and analysis of randomised trials. Br J Sports Med 52, 553-555.

29. Mansournia MA, Higgins JP, Sterne JA, et al. (2017) Biases in randomized trials: a conversation between trialists and epidemiologists. Epidemiology 28, 54-59.

30. Greenland S \& Mansournia MA (2015) Limitations of individual causal models, causal graphs, and ignorability assumptions, as illustrated by random confounding and design unfaithfulness. Eur J Epidemiol 30, 1101-1110.

31. Stranges S, Marshall JR, Natarajan R, et al. (2007) Effects of long-term selenium supplementation on the incidence of type 2 diabetes: a randomized trial. Ann Intern Med 147, 217-223.

32. Galan-Chilet I, Grau-Perez M, De Marco G, et al. (2017) A gene-environment interaction analysis of plasma selenium with prevalent and incident diabetes: The Hortega study. Redox Biol 12, 798-805.

33. Laclaustra M, Navas-Acien A, Stranges S, et al. (2009) Serum selenium concentrations and diabetes in U.S. adults: National Health and Nutrition Examination Survey (NHANES) 2003-2004. Environ Health Perspect 117, 1409-1413.

34. Cold F, Winther KH, Pastor-Barriuso R, et al. (2015) Randomised controlled trial of the effect of long-term selenium supplementation on plasma cholesterol in an elderly Danish population. Br J Nutr 114, 1807-1818

35. Boskabadi H, Maamouri G, Rezagholizade Omran F, et al. (2012) Effect of prenatal selenium supplementation on cord blood selenium and lipid profile. Pediatr Neonatol 53, 334-339.

36. Safaralizadeh R, Kardar GA, Pourpak Z, et al. (2005) Serum concentration of selenium in healthy individuals living in Tehran. Nutr J 4, 32.

37. Rafraf M, Mahdavi R \& Rashidi MR (2008) Serum selenium levels in healthy women in Tabriz, Iran. Food Nutr Bull 29, $83-86$.

38. Rayman MP (2008) Food-chain selenium and human health: emphasis on intake. Br J Nutr 100, 254-268.

39. Gao H, Hagg S, Sjogren P, et al. (2014) Serum selenium in relation to measures of glucose metabolism and incidence of type 2 diabetes in an older Swedish population. Diabet Med 31, 787-793. 
40. Wei J, Zeng C, Gong QY, et al. (2015) The association between dietary selenium intake and diabetes: a crosssectional study among middle-aged and older adults. Nutr J 14, 18.

41. Zhou J, Huang K \& Lei XG (2013) Selenium and diabetes evidence from animal studies. Free Radic Biol Med 65, 1548-1556.

42. Alizadeh M, Safaeiyan A, Ostadrahimi A, et al. (2012) Effect of L-arginine and selenium added to a hypocaloric diet enriched with legumes on cardiovascular disease risk factors in women with central obesity: a randomized, double-blind, placebocontrolled trial. Ann Nutr Metab 60, 157-168.

43. Kaur HD \& Bansal MP (2009) Studies on HDL associated enzymes under experimental hypercholesterolemia: possible modulation on selenium supplementation. Lipids Health Dis $\mathbf{8}, 55$.

44. Kuboki K, Jiang ZY, Takahara N, et al. (2000) Regulation of endothelial constitutive nitric oxide synthase gene expression in endothelial cells and in vivo : a specific vascular action of insulin. Circulation 101, 676-681.

45. van Herpen NA \& Schrauwen-Hinderling VB (2008) Lipid accumulation in non-adipose tissue and lipotoxicity. Physiol Behav 94, 231-241.

46. Li YB, Han JY, Jiang W, et al. (2011) Selenium inhibits high glucose-induced cyclooxygenase-2 and P-selectin expression in vascular endothelial cells. Mol Biol Rep 38, 2301-2306.

47. Brigelius-Flohe R, Banning A, Kny M, et al. (2004) Redox events in interleukin-1 signaling. Arch Biochem Biophys $\mathbf{4 2 3}$, 66-73.

48. Alehagen U, Lindahl TL, Aaseth J, et al. (2015) Levels of sP-selectin and hs-CRP decrease with dietary intervention with selenium and coenzyme Q10 combined: a secondary analysis of a randomized clinical trial. PLOS ONE 10, e0137680.

49. Vunta H, Davis F, Palempalli UD, et al. (2007) The antiinflammatory effects of selenium are mediated through 15deoxy-delta12,14-prostaglandin $\mathrm{J} 2$ in macrophages. $J$ Biol Chem 282, 17964-17973.

50. Vunta H, Belda BJ, Arner RJ, et al. (2008) Selenium attenuates pro-inflammatory gene expression in macrophages. Mol Nutr Food Res 52, 1316-1323.

51. Brodska H, Valenta J, Malickova K, et al. (2015) Biomarkers in critically ill patients with systemic inflammatory response syndrome or sepsis supplemented with high-dose selenium. J Trace Elem Med Biol 31, 25-32.

52. Zhu X \& Lu Y (2012) Selenium supplementation can slow the development of naphthalene cataract. Curr Eye Res 37, 163-169.

53. Gullestad L \& Aukrust P (2005) Review of trials in chronic heart failure showing broad-spectrum anti-inflammatory approaches. Am J Cardiol 95, 17C-23C.

54. Amir O, Paz H, Rogowski O, et al. (2009) Serum oxidative stress level correlates with clinical parameters in chronic systolic heart failure patients. Clin Cardiol 32, 199-203.

55. Bleys J, Navas-Acien A \& Guallar E (2008) Serum selenium levels and all-cause, cancer, and cardiovascular mortality among US adults. Arch Intern Med 168, 404-410.

56. Akbaraly NT, Arnaud J, Hininger-Favier I, et al. (2005) Selenium and mortality in the elderly: results from the EVA study. Clin Chem 51, 2117-2123.

57. Ray AL, Semba RD, Walston J, et al. (2006) Low serum selenium and total carotenoids predict mortality among older women living in the community: the women's health and aging studies. J Nutr 136, 172-176. 